\title{
Role of platelets in blood-biomaterial interactions
}

\author{
G H R RAO* and THOMAS CHANDY \\ Biomedical Engineering Institute, Academic Health Centre, PB 609 UMHC, University of Minnesota, 420 Delaware \\ St. SE. Minneapolis, MN 55455, USA
}

\begin{abstract}
Over 2 million cardiovascular procedures are performed annually in the United States. Every one of these procedures requires some period of contact with blood with several different biomaterials used in the manufacture of assist devices or implant devices. In view of the increasing importance of the biomaterials in clinical practice, it would be timely to review briefly physicochemical characterization as well as biological evaluations. Blood compatibility encompasses a variety of events associated with blood interaction with the biomaterials used in various procedures. Two separate coagulation mechanisms are involved (arterial and venous) depending upon the flow characteristics. At least three interacting factors modulate normal hemostasis and the pathogenesis of thromboembolic events. They are the state of activation of coagulation cascade, circulating levels of thrombin and fibrinogen and relative activity of platelets. In this overview we discuss the current concepts on the role of platelets in blood-biomaterial interactions.

When blood contacts a biomaterial surface a variety of blood components interact with the surface. Some of the key players in platelet activation on biomaterial surface include fibrinogen and von Willebrand factor. Currently available antiplatelet drugs effectively block aggregation and secretion induced by physiological agonists such as epinephrine, adenosine diphosphate and thromboxane in suspension. However, they do not prevent platelet interaction on biomaterial surfaces. Mechanisms involved in platelet activation in suspension and on surfaces as well as the pharmacology of newer antiplatelet drugs will be discussed.
\end{abstract}

Keywords. Platelet adhesion; protein adsorption; biomaterials; surface induced platelet activation; fibrinogen; platelet-surface receptors; antiplatelet drugs.

\section{Introduction}

The adhesion of circulating platelets at sites of injury on the vessel wall where subendothelium, in particular collagen, is exposed to the flowing blood, is known to be an important step in a sequence of events leading to the formation of a hemostatic plug (Mustard and Packham 1975). With the development of prosthetic devices and artificial organs, the study of platelet adhesion and thrombus formation on synthetic polymer surfaces has become very important in assessing the compatibility of biomaterials to blood. A clear understanding of the blood interactions with artificial surfaces would promote a better utilization of existing materials and the development of new materials with improved properties.

\subsection{Contribution of platelets to thrombus formation}

1.1a Platelet morphology, physiology and function: Platelets are the disc-shaped fragments of bone marrow megakaryocytes circulating in blood. They are about $250,000 \pm 80,000$ per $\mu$ l. They have a life span of $8-10$ days and play a critical role in the recognition of vascular injury, formation of effective hemostatic plugs, retraction of clots and wound healing. However, when hyperactive,

\footnotetext{
*Author for correspondence
}

they can initiate events leading to clinical complications associated with cardiovascular and cerebrovascular diseases. Although completely nonsticky in the resting state, they become sticky with the slightest stimulation. The degree of activation depends on the strength of the stimulus and the type of signal generated by ligandreceptor interaction. Four well developed stages of activation are recognized: (i) development of stickness, (ii) changes in cell shape, (iii) contraction and release of granule contents and (iv) irreversible aggregation.

Platelet plasma membranes contain transmembrane proteins as well as glycoproteins. Glycoproteins serve as the receptors for agonist/surface-mediated stimuli initiating platelet activation. Platelets contain integrin and nonintegrin domains in their plasma membranes. Integrins are transmembrane glycoproteins with alpha and beta units coupled noncovalently (GPIIb-IIIa, GPIa-IIa, GPIcIIa), They participate in both cell-cell and cell-matrix interactions. In addition, there are nonintegrin glycoprotein domains on platelets capable of interacting with acromolecules such as vWF and collagen (GPIV, GPIb).

$1.1 \mathrm{~b}$ Role of platelets in thrombosis: Circulating platelets rapidly adhere to the subendothelial matrix, become activated, adhere, spread, release the contents of various storage organelles and become cohesive, leading to 
formation of an aggregate or plug of platelets that prevents blood loss from the damaged vessel. The initial event in hemostasis and thrombosis is the contact adhesion of blood platelets to vessel wall subendothelial matrix at high shear flow (Baumgartner et al 1976). Hemostatic plugs and arterial thrombi are largely composed of platelet aggregates adherent to the injury site, with fibrin in and around the platelets acting to stabilize the plug or thrombus (Packham and Mustard 1984). Platelets that interact with the subendothelium change from their normal disc shape to a more round form, extend pseudopods, and become adherent to the surface (Chesterman and Berndt 1986). Alternatively, they may release their granule contents into the surrounding medium, which include ADP, ATP, calcium, serotonin, platelet factors, $\beta$-thromboglobulin, fibrinogen, fibronectin etc. (Salzman et al 1977).

There are several ways in which platelets can contribute towards the coagulation process. First, the platelet surface protects active clotting factors from inactivating by their natural inhibitors (Jackson and Nemerson 1980). Second, platelets contribute through aggregation, during which platelet phospholipids (platelet factor 3) are made available and stimulate activation of factor $\mathrm{X}$ and the conversion of prothrombin to thrombin (Chesney et al 1982). Finally, during the release reaction fibrinogen, platelet factor 4 and a variety of proaggregatory compounds are made available. The amount of released fibrinogen is small in relation to plasma fibrinogen level (White and Heptinstall 1978). However, the exact role of this newly released fibrinogen in platelet/biomaterial interaction and the subsequent promotion of thrombus growth is not clear.

\subsection{Platelet adhesion on biomaterials}

Activation of platelets by contact with artificial surfaces is a key event in the thromboembolic complications of prosthetic devices in contact with the blood (Park and Park 1989). It is known that a film of plasma protein adsorbs on artificial materials exposed to blood and that this event proceeds interaction of the surface with blood cells (Feuerstein and Sheppard 1993). Platelet adhesion to surfaces occurs in various steps including initial attachment, spreading, release of granule contents, and platelet aggregation (Friedman et al 1970). These changes are collectively termed surface-induced platelet activation. During the process of platelet activation on surfaces, platelets continuously change shape while the platelet ventral membrane makes transient surface contacts. Platelet adhesion and shape change are mediated by cytoskeletal reorganization, as observed by high voltage electron microscopy (Park and Park 1989). It is also believed that platelets release their granule contents into the surrounding medium, thus providing proaggregatory compounds for the promotion of platelet aggregate formation.

Platelets are seen to adhere rapidly to various surfaces upon contact with blood, either as a monolayer or as aggregates and this process is influenced by a combination of factors including surface smoothness, surface charge, wettability, surface tension and the flow conditions (Mohammad et al 1974). Baier et al (1985) studied the effect of surface energetics of substrates towards platelet attachment, their spreading and correlated towards bloodcompatibility. He suggested that minimal platelet spread areas were found on substrata with critical surface tension between 20 and 30 dynes $/ \mathrm{cm}$ and increased spreading and morphological changes occurred on substrata of both higher and lower critical surface tension. So the adhesion and spreading of platelets to artificial substrates have upmost importance to predict the subsequent process of thrombus formation at the interface.

1.2a Role of plasma proteins on platelet adhesion: Immediately following implantation, the prosthetic surface is exposed to flowing blood, and plasma proteins are adsorbed on the surface of the artificial matrix. The proteins such as albumin, fibrinogen and globulins are rapidly deposited on artificial surfaces and are partially replaced over time by high-molecular weight kininogen, and hageman factor (Vroman et al 1982). Ziats et al (1990) have shown that fibronectin, hemoglobin and von Willebrand factor are also deposited on artificial surfaces exposed to flowing blood. The platelet-surface interactions can be described as consisting of three consecutive events (Difazio 1994): (I) An initial platelet-to-surface adhesion occurs between platelet surface receptors and adsorbed matrix proteins. The information provided by the interactive domains of cell matrix components dictate degree of activation. For instance, laminin induces only focal adhesion, whereas, fibronectin promotes spreading and collagen supports aggregation and secretion. (II) This adhesion results in platelet activation, with subsequent degranulation and release of platelet activating factors. (III) Thrombus formation results from the deposition of additional blood elements and recruitment of platelets.

1.2b Role of fibrinogen: Fibrinogen appears to be an important component of the adsorbed protein film and may be an essential player in the early events of platelet activation adsorbing preferentially on many surfaces in a concentration relatively higher than in the bulk plasma and participating in the platelet response to the prosthetic surface (Brash and Suniyal 1979). An early feature of activation of platelets by soluble agonists such as ADP or thrombin is exposure of specific membrane glycoproteins (GPIIb-IIIa), to which fibrinogen molecule bind with high affinity (Bailly et al 1996). It should be noted that fibrinogen dissolved in plasma does not induce 
platelet aggregation but rather acts as a cofactor in the process, once platelet GPIIb/IIIa receptors are activated. Fibrinogen normally circulates in peaceful coexistence with unactivated platelets without any obvious interaction (Peerschke et al 1980).

It is claimed that platelets have membrane receptors of low affinity for fibrinogen, which would permit reversible interactions at physiologic fibrinogen concentrations (Bailly et al 1996). The prevailing view is that it is only in the 'activated' platelet (i.e. the platelet stimulated by agonists such as thrombin or ADP) that fibrinogen forms a detectable complex with its glycoprotein membrane receptors, which are 'masked' or otherwise unavailable in intact nonactivated circulating platelets (Marguerie et al 1979). Surface bound fibrinogen is involved in platelet adhesion to surfaces by bridging the gap between platelet and surface in a manner analogous to its action in platelet-platelet aggregates. It has been suggested that fibrinogen molecules adsorbed on artificial surfaces undergo conformational changes and interact with unactivated platelets to promote their adhesion on the surface. They may even be responsible for the progression from platelet adhesion to aggregation and secretion (Salzman et al 1977).

Vroman et al (1980) extensively studied the initial phase of blood-surface interactions. They have postulated that on hydrophilic surfaces (e.g. Glass), Fg is deposited within seconds along with traces of high molecular weight kininogen (HMWK) and factor XII. Then more HMWK and factor XII arrive and displace Fg. Platelets adhere most, where fibrinogen remains. Thus, Fg plays a key role in platelet-surface attachment and subsequent thrombus formation.

1.2c Role of other cell adhesive proteins: The platelet receptor-endothelial matrix protein interactions have been well characterized. Under high shear conditions, this attachment is mediated by the binding of von Willebrand Factor (vWF) to the glycoprotein receptor GPIb-IX (Fressinaud et al 1988). Secondary attachment between platelets results in thrombus formation, which is caused by $\mathrm{Fg}$ or vWF-mediated crosslinking of GPIIb-IIIa receptors on adjacent platelets. Several other matrix protein ligands such as laminin, fibronectin, collagen and their associated platelet receptors have been shown to contribute to platelet adhesion and aggregation to the subendothelium. While the receptors involved in platelet adhesion to the subendothelium have been studied extensively, the receptors involved in platelet adhesion to prosthetic materials are less well identified. The biomaterials adsorb a layer of plasma proteins on their surface upon exposure to blood. The content of this protein layer varies with time and induces $\mathrm{Fg}, \mathrm{Ig} \mathrm{G}$, Fn, vWF and vibronectin (Dafazio et al 1994) etc for which there are specific surface receptors on platelet surface (Andrews and Fox 1991).
The platelet attachment and spreading on glass and various protein coated glass, under shear $\left(1000 \mathrm{~s}^{-1}\right)$ were monitored using washed platelets, platelet rich plasma (PRP) and whole blood via fluorescence optimas imaging system and software (Chandy et al 1998). The studies suggested that the platelet adhesion and spreading was higher with $\mathrm{Fg}, \mathrm{Fn}$ and vWF coated glass and least on albuminated surfaces. The binding of $\mathrm{Fg}$ and other platelet adhesive proteins to a surface may induce conformational changes in the proteins, which could result in higher affinity binding of these proteins with platelet adhesion receptors. Thus the variations in platelet adhesion and spreading to albuminated glass and other adhesive protein coated glass may be due to the status and availability of platelets and other protein molecules in solution at the interface. Goodman et al (1993) have suggested that GPIIb/IIIa may be involved in the spreading process of platelet-fibrinogen interaction. Adhesive plasma proteins in general possess RGD binding domain which interacts with GPIIb-IIIa ( $\mathrm{Fg}, \mathrm{Fn}$ and perhaps von Willebrand factor), and induce actin polymerization and receptorcytoskeleton tethering, as occurs when Fg binds GP1 b111a. Based upon these observations, it is reasonable to speculate how integrin receptors could influence subsequent platelet spreading on synthetic surfaces. The cells spread differently on material surfaces, which had been modified with denatured and conformationally altered plasma proteins. Because platelet surfaces are largely glycoprotein-rich, one possibility is that adsorption-induced alterations in the secondary structure of these proteins somehow leads to cellular response. The plasma proteins like Fg and vWF are unable to bind their respective receptors when in solution, yet once adsorbed to surfaces the proteins become 'active' and are capable of receptor-ligand coupling.

Fibrinogen adsorption from plasma and platelet attachment from blood to various protein coated glass substrates are measured as a function of exposure time, under shear $\left(1000 \mathrm{~s}^{-1}\right)$. It appears from figure 1 that $\mathrm{Fg}$-surface binding is maximum on fibrinogen precoated glass, followed by other platelet adhesive proteins and least on albuminated surfaces. However, it is likely that loosely

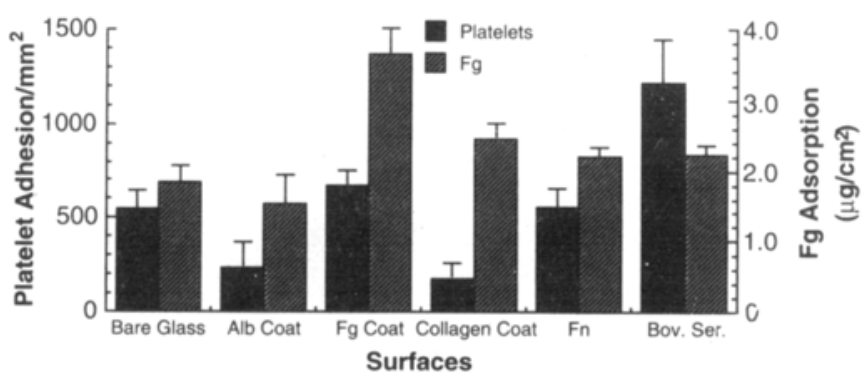

Figure 1. Fibrinogen adsorption from plasma vs platelet adhesion from blood to protein coated glass for $15 \mathrm{~min}$ exposure at $1000 \mathrm{~s}^{-1}$ shear, $\mathrm{Fg}$ and $\square$ platelets. 
adsorbed albumin molecules are displaced by plasma to enhance its surface concentration. These results suggest that there is no direct correlation with $\mathrm{Fg}$ surface attachment and platelet adhesion. Not all bound Fg may be in a state in which platelet receptors can interact. Platelet GPIIb/IIIa can only recognize the bound fibrinogen that has undergone appropriate conformational changes and has the RGD site available for interaction with the receptor (Kloczewiak et al 1989). Studies of Lindon et al (1986) have shown that platelet adhesion is related to the amount of antibody-recognizable fibrinogen domains rather than total amount of $\mathrm{Fg}$ on biomaterials. Thus, it is reasonable to speculate that the surface properties affects the conformation of adsorbed $\mathrm{Fg}$ and other platelet-adhesive protein molecules in a variety of ways and thereby influences subsequent platelet adhesion and spreading to a different degree.

\subsection{Effect of shear on platelet-surface interaction}

The in vitro study of the hemocompatibility of biomaterials requires the consideration of many parameters, static or dynamic contact, flow rate, wall shear rate, form of biomaterial to be tested, pathway to consider (platelet adhesion, platelet activation, complement activation, contact phase activation etc) and duration of contact (Rhodes et al 1996). It has previously been demonstrated that hemodynamic circumstances play a significant role in determining localization, growth and fragmentation of thrombi and platelet adhesion in vivo, and that flow rate controls platelet transport to a surface and their adhesion (Goldsmith 1972). The effect of flow rate on cell adhesion has been discussed in detail by several researchers in terms of shear rate (Goldsmith 1972; Absolom et al 1984-1985). Generally increased flow rate diminished platelet adhesion until some critical shear rate is reached at which erythrocyte hemolysis occurs. The release of hemoglobin then appears to promote increased cell adhesion. Fluid mechanical trauma, low to moderate levels of shear stress $\left(100\right.$ to $\left.300 \mathrm{dyn} / \mathrm{cm}^{2}\right)$ can produce altered polymorphonuclear neutrophil leukocyte morphology and function. Thus the shear related changes include an increased adhesion, aggregation and cell lysis, moderate decrease in chemiluminescence accompanying phagocytosis, and substantial loss of lysosomal enzymes. These changes also cause an increased platelet-platelet sticking and the formation of microemboli.

\subsection{Platelet activation and morphological changes}

Platelet adhesion to surfaces occurs in various steps including initial attachment, spreading and release of granule contents and platelet aggregation. During the process of such activation sequences on surfaces, platelets continuously change shape while the platelet ventral membrane makes transient surface contacts. Platelet adhesion and shape change are mediated by cytoskeletal

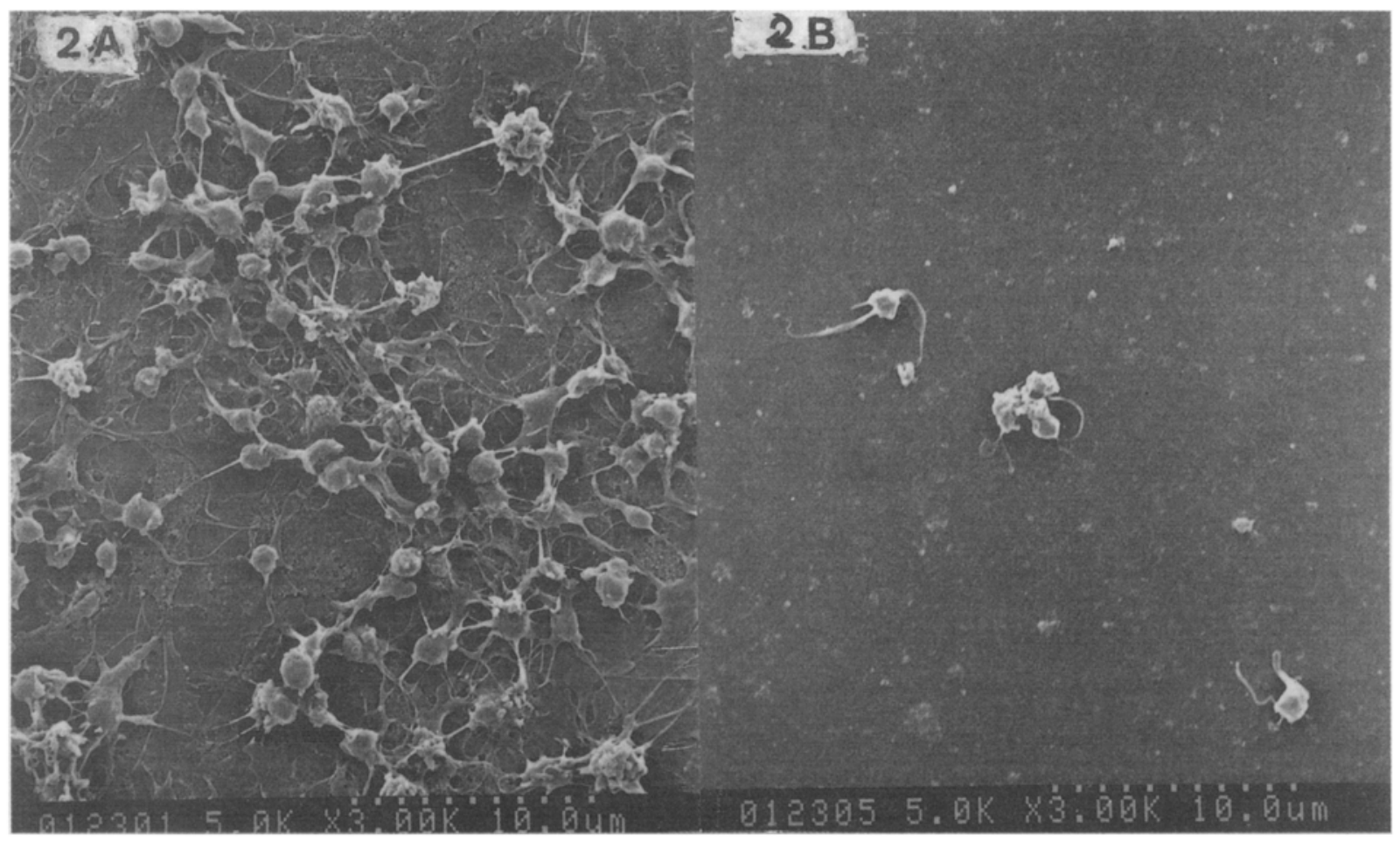

Figure 2. Scanning electron micrographs of adhered platelets to (A) polycarbonate and (B) polyelectrolyte coated polycarbonate, on exposure to PRP for $1 \mathrm{~h}$. 
reorganization as observed by high voltage electron microscopy (Park and Park 1989). The reorganization of the platelet cytoskeleton, upon surface activation, is critical for pseudopod extension and retraction, centripetal movement of the platelet fibrinogen receptors, and the centralization and secretion of granules. Scanning electron microscopy (SEM) has been used to visualize specific regions of intimate contact between the ventral surface of adherent cells and an underlying surface. Polycarbonate and poly propylene surfaces were coated with sulphonated polyelectrolytes (SPE) to improve their blood compatibility. Figure 2 indicates the scanning electron micrographs of the adhered platelets (from PRP, $1 \mathrm{~h}$ ) on polycarbonate and SPE modified polycarbonate. The platelet adhesion and spreading was higher with bare polymer (figure $2 \mathrm{~A}$ ), which had substantially reduced interaction due to SPE (figure 2B) treatments. The adhesion and spreading of platelets (from PRP, $2 \mathrm{~h}$ ) on poly propylene was similarly inhibited by pretreatment with sulphonated polyelectrolytes (figure 3 ). Regardless of treatment with SPE, all platelets adherent to polycarbonate and poly propylene were pseudopodial (figures 2 and 3 ).

Polyelectrolytes are synthetic analogues, having sulfamate and carboxylic groups arranged in a steric manner as that of heparin. These synthetic polyelectrolytes are employed on biomaterials for improving their biocompatibility and have shown antiplatelet and anticogulant activity (Sharma et al 1984-1985). The protein surface binding in presence of polyelectrolyte has been investigated, from a protein mixture, using polyacrylamide gel electrophoresis (Chandy and Sharma 1987). The synthetic polyelectrolyte has enhanced the surface binding of albumin and has reduced the fibrinogen and $r$-globulins. The observation that SPE reduces platelet-surface attachment may partly be due to the reduced availability of platelet receptors for $\mathrm{Fg}$ on the modified material.

\subsection{Pharmacological approaches to inhibit platelet-surface attachments}

In clinical applications, foreign surfaces represent only one of the factors influencing the blood/biomaterial interactions. These interactions will also be influenced by the presence of antithrombotic agents, the blood condition and the nature of the application. It is important in the clinical set up to remember that blood-material interaction depends not only on the material being used but also on constituents of the blood which may alter in disease states, i.e, the blood may be more hypercoagulable and platelets may be activated (Courtney and Forbes 1994). Many of the platelet reactions which have been previously described may be altered by appropriate antithrombotic therapy, in particular by heparin, warfarin and fibrinolytic agents and it is a combination of these factors that determines the long-term viability of the procedure which is being undertaken.

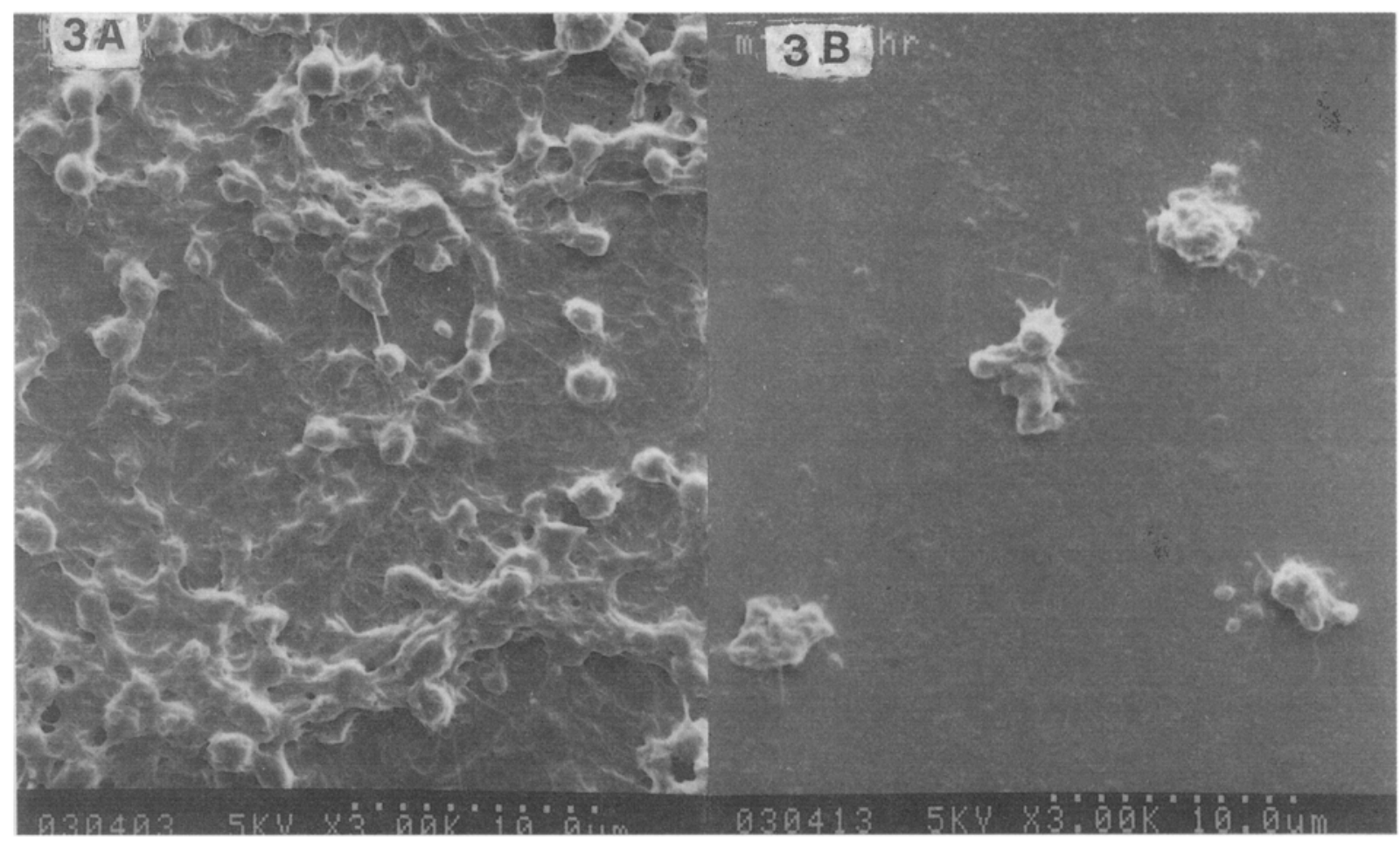

Figure 3. Scanning electron micrographs of adhered platelets to (A) polypropylene and (B) polyelectrolyte coated polypropylene, on exposure to PRP for $2 \mathrm{~h}$. 
Currently available antiplatelet drugs effectively block platelet aggregation and secretion induced by physiological agonists such as epinephrine, adenosine diphosphate and thromboxane in suspension (Rao and Rao 1994). These drugs exert their effect by blocking several different activation signalling mechanisms. Some of the known compounds that modulate platelet function include: inhibitors of arachidonic acid metabolism (nonsteroidal anti-inflammatory drugs and thromboxane synthetase inhibitors), drugs that alter membrane phospholipid composition (omega 3 fatty acids), stimulators of adenyl cyclase and guanylyl cyclase $\left(\mathrm{PGE}_{1}, \mathrm{PGI}_{2}, \mathrm{PGD}_{2}\right.$, nitroglycerine, nitropruside), phosphodiesterase inhibitors (dipyridamole and methyl xanthines) and calcium antagonists (verapamil, nifedipine, diliazem). Most of these drugs are not totally effective on preventing platelet interaction on biomaterial surfaces.

Earlier studies have indicated that certain vitamins, antibiotics, antihypertensive drugs, antiplatelet agents etc can modulate the protein-surface binding and subsequent cellular attachment towards an artificial substrate (Chandy and Sharma 1987, 1988). It seems vitamin $B_{6}, C, E$ combinations of aspirin-vit. C, certain antibiotics etc influence the fibrinogen-surface concentration or enhance the albumin surface-binding to variable degrees. This may be one of the reasons for a reduced platelet-surface binding in presence of these agents. So it is possible that the antiplatelets arriving as mediators at the interface may alter the sequence of proteins deposited or displaced by plasma; and favour albumin binding in several cases. Platelets that arrive in the later stages find $\mathrm{Fg}$ to adhere. In summary, the clinical use of artificial surfaces in contact with blood leads to a response influenced by the material/device, antithrombotic agents, blood condition and application.

\section{Conclusion}

The increasing use of biomaterials in cardiovascular applications underlines the need for a better understanding of the interactions of blood with foreign surfaces. Platelet adhesion, shape change and secretion of granules upon exposure to biomaterial surfaces in flowing blood are the initial and critical reactions that facilitate thrombus formation and growth. The activation-independent and dependent integrin receptors-glycoproteins (GPIc-IIa, GPIIb-IIIa) are involved in platelet adhesion and thrombus growth on biomaterial interfaces through interactions with fibrinogen, fibronectin, vWF and other adhesive proteins. Further studies are essential to understand the complex chemical changes in polymers, in vivo effects due to surface modifications, effect of antithrombotic agents used to supress platelet-surface interaction and also the coagulation cascade to obtain long-term acceptability of new clinical devices for biomedical applications.

\section{Acknowledgements}

The authors thank Dr Yuanyuan Zhang for some of the SEM photomicrographs and Departments of Laboratory Medicine and Pathology and Biomedical Engineering Institute for financial support.

\section{References}

Absolom D R, Zingg W, Van Oss C J and Newmann A W 1984-1985 Biomater. Med. Devices. Artif. Organs 12235

Andrews R K and Fox J E B 1991 Curr. Opin. Cell. Biol. 2 894

Baier R E, Depalma V A, Goupil D W and Cohen E $1985 \mathrm{~J}$. Biomed. Mater. Res. 191157

Bailly A L et al $1996 \mathrm{~J}$. Biomed. Mater. Res. 30101

Baumgartner H R, Muggli R, Tschopp T B and Turitto V T 1976 Thromb. Haemost. 35124

Brash J L and Suniyal S 1979 J. Polym. Sci. 66377

Chandy T and Sharma C P 1987 Polym. Plast. Tech. Engg. 26 143

Chandy T and Sharma C P 1988 Artif. Organs 12143

Chandy T, Mooradian D L and Rao G H R $1998 \mathrm{~J}$. Biomater. Appl. 1346

Chesney C M, Pifer D D, Byers L W and Muirhead E E 1982 Blood 59582

Chesterman C N and Berndt M C 1986 Clin. Haematol. 15 323

Courtney J M and Forbes C D 1994 Br. Med. Bull. 4966

Difazio L T, Stratoulias C, Greco R S and Haimovich B 1994 J. Surg. Res. 57133

Feuerstein I A and Sheppard J I 1993 Biomaterials 14137

Fressinaud E, Baruch D, Girma J P, Sakariassen K S, Baumgartner H R and Meyer D $1988 \mathrm{~J}$. Lab. Clin. Med. 11258

Friedman L I, Liem H, Grabowski E F, Leonard E F and McCord C W 1970 Trans. Am. Soc. Artif. Intern. Organs 16 63

Goldsmith H L 1972 Prog. Haemost. Thromb. 197

Goodman S L, Cooper S L and Albrecht R M 1993 J. Biomed. Mater. Res. 27683

Jackson C M and Nemerson Y 1980 Ann. Rev. Biochem. 49 765

Kloczewiak M, Timmons S, Bednarek M A, Sakon $M$ and Hawiger J 1989 Biochemistry 282915

Lindon J N, McManama G, Kushner L, Merrill E W and Salzman E W 1986 Blood 68355

Marguerie G A, Plow E F and Edgungton T S $1979 \mathrm{~J}$. Biol. Chem. 2545357

Mohammad S F, Hardison M D, Glenn C H, Morton B D, Bolan J C and Mason R G 1974 Haemostasis 3257

Mustard J F and Packham M A 1975 Thromb. Diathes. Haemorrh. 33444

Packham M A and Mustard J F 1984 in Blood platelet function and medicinal chemistry (ed.) A Lasso (New York: Elsevier Biomedical) pp 61-128

Park K and Park H 1989 Scan. Microscopy Suppl., 3137

Peerschke E I, Zucker M B, Grant R A, Egan $\mathbf{J} \mathbf{J}$ and Johnson M M 1980 Blood 55841 
Rao G H R and Rao A 1994 Indian. J. Physiol. Pharmacol. 3869

Rhodes N P, Kumary T V and Williams D F 1996 Biomaterials 171995

Salzman E W, Lindon J, Brier D and Merrill E W 1977 Ann. N. Y. Acad. Sci. 283114

Sharma C P, Chandy T and Ashalatha P V 1984-1985 Biomater. Med. Devices. Artif. Organs 12215
Vroman L, Adams A L, Fischer G C and Munoz P C 1980 Blood $\mathbf{5 5} 156$

Vroman L, Adams A L, Fischer G C. Munoz P C and Stanford M 1982 Adv. Chem. Ser. 199266

White A M and Heptinstall S 1978 Br. Med. Bull. 34 123

Ziats N P, Pankowsky D A, Tierney B P, Ratnoff O D and Anderson J M 1990 J. Lab. Clin. Med. 116687 